\title{
The disposable soma theory of aging in reverse
}

\author{
Cell Research (2014) 24:7-8. doi:10.1038/cr.2013.148; published online 5 November 2013
}

In a recent paper in Nature, Ermolaeva et al. uncover a systemic response to DNA damage in germ cells that protects somatic tissues, providing mechanistic insight into the bidirectional communication between germ line and soma.

A general reset of all molecular processes during reproduction enables every generation to be born new. Otherwise defects in cellular fidelity, which decrease the lifespan of the animal by a mere second or minute, could accumulate over successive generations and push the species to extinction. In 1889 August Weismann put forth a revolutionary idea that the germ line was solely responsible for inheritance of traits and the soma contributed nothing. This theory introduced the notion that the germ line possessed characteristics to ensure immortality and not to lose a second or minute for each generation. These ideas were formalized by Thomas Kirkwood in the disposable soma theory of aging [1]. For the last several decades the aging research community has witnessed numerous studies supporting the hypothesis that resources are allocated from the soma to the germ line under conditions of stress and aging in order to ensure the fitness of the ensuing generation. However, work from Ermolaeva et al. [2] provided evidence that this is not a unidirectional path going from soma to germ line. In fact, certain stresses when applied to the germ line can protect the soma. This observation has initiated a re-evaluation of Weismann's hypothesis.

Ermolaeva et al. uncover a systemic stress response to DNA damage that acts through an innate immune response pathway. Using the nematode C. elegans, they demonstrate that DNA damage occurring in the germ line, representing the worm's stem cell niche, initiates protective stress signals that propagate to somatic tissues (Figure 1). In turn, these stress-responsive signals act in somatic tissues to bolster protein homeostasis through the increased function of the ubiquitin/ proteosome system. The protective systemic response to DNA damage initiated in germ cells is termed "germ line DNA-damage-induced systemic stress resistance" or GDISR.

These observations represent a growing body of research highlighting the importance of trans-cellular signaling. In these paradigms, a master tissue or cell type can sense perturbations within its own cellular environment and mediate organism-wide protection in other tissues. In C. elegans, the nervous system has emerged as an important tissue through which systemic signaling events can occur. Neurons appear sensitive to various disruptions in protein homeostasis and are capable of generating signals that are secreted to initiate protective transcriptional changes in distal tissues. Interestingly, the nature of the stress, which is sensed by the neuron, appears to generate different signals. Unfolded protein stress in different sub-cellular compartments of the neuron, including mitochondria [3], endoplasmic reticulum [4] and cytoplasm [5], possesses unique signaling properties that transmit the respective stress response information to distal tissues. Thus, organisms can sense cellular dysfunction in a few cells and quickly initiate protective transcriptional programs throughout the animal.

Trans-cellular signaling in nematodes primarily involves the nervous system as the essential tissue to sense stress and initiate protective responses throughout the animal. Ermolaeva et al. provide insight into the trans-cellular signaling network originating in germ cells. The germ line tissue houses the only meoiotic cells in the adult worm and is responsible for progeny production. Thus stress or dysfunction within germ cells can compromise reproductive fidelity and have detrimental effects on subsequent worm generations. Perhaps more so than any other response, genotoxic stress in germ cells can result in DNA damage and thereby introduce numerous errors or mutations within the progeny's genome. Thomas Kirkwood proposed that animals can shunt energy and resources away from somatic tissues and toward the germ line to maintain reproductive integrity [1]. In support of his disposal soma theory, ablation of germ cells yields longer-lived, more stressresistant animals $[6,7]$. Yet the mechanism of how germ cells and somatic tissue ration their resources remains unclear.

Ermolaeva et al. provide important insight into the communication between germ line and soma. They observe that the germ line is a key tissue for sensing DNA damage and subsequently communicating to somatic tissues under genotoxic stress. This signaling cascade relies on an innate immune response in germ cells to transmit protective programs to the 


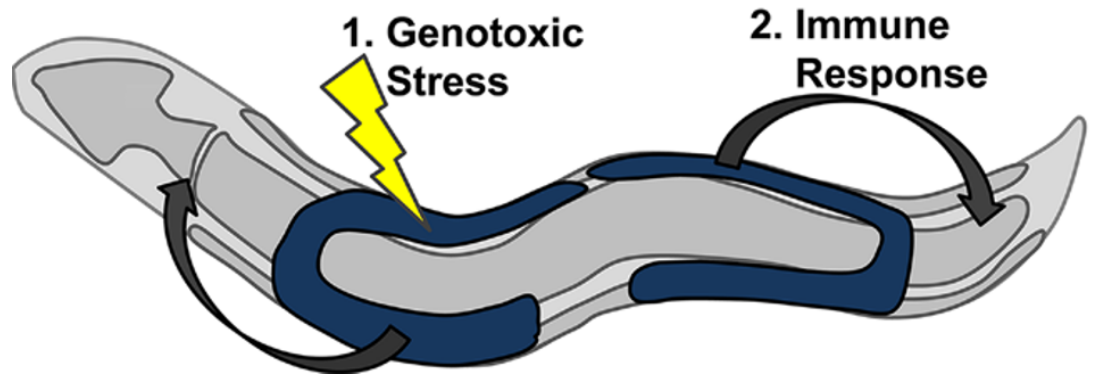

Figure $1 \mathrm{Germ}$ line in the nematode protects somatic tissue through innate immune response under genotoxic stress. The model represents an adult nematode in which somatic tissue is depicted in gray and the germ line is depicted in blue. 1. Genotoxic stress causes DNA damage in the germ cells. 2. Germ cells transmit stress signal to the peripheral somatic tissues. Upon receipt of the signal, the soma activates protective clearance mechanisms to remove damaged and misfolded proteins.

soma. Upon receipt of the genotoxic stress signal, the somatic tissues increase ubiquitin/proteasome function and accelerate protein turnover in the cells. In this manner, somatic tissues can readily remove misfolded proteins resulting from error-ridden translation of messenger RNA and promote healthier, more stress-resistant cells. Although the germ cell stress signal was not clearly defined, it may act through the proteasomal capping subunit, RPN-6, which has been shown to influence proteasomal function [8].

Ermolaeva et al. delineate this transcellular signaling pathway by implicating molecular players in the initiating germ cells. This provides the putative signaling mechanism involved in stress signal transmission and helps highlight the molecular machinery in the receiving somatic tissues that executes the protective program. Upon DNA damage, the resident germ line ERK mitogen activating protein kinase, MPK-1, initiates a protective cascade that ul- timately results in the secretion of an extracellular signal from the germ line to the soma. Upon receipt of the stress signal, the soma increases protein turnover through the ubiquitin/proteasome system. However, it is unclear how DNA damage is sensed by MPK-1 in germ cells and what molecular components of the ubiquitin/proteasome system are altered by receipt of the signal.

Had modern-day tools been available, would August Weismann have re-evaluated his theory that germ cells were solely responsible for inheritance of traits and the soma contributes nothing? Ermolaeva et al. begin to uncover the bidirectional allocation of resources between germ line and soma and implicate the innate immune response as a trans-cellular signaling mechanism by which the germ line protects the soma. This model adds a new level of complexity to the innate immune response that is traditionally characterized as a transcriptional response to pathogen invasion of the host. The potential for protection from genotoxic stress has major implications in the biomedical field. In mammals, the innate immune response has long been known to mediate inflammation. However, it is possible that DNA damage in humans might enhance tissue maintenance prior to chronic inflammation. A growing body of evidence has started to link the innate immune response in mammalian models to DNA damage [9] as well as tissue maintenance and regeneration [10]. Further research will uncover whether the inflammatory response is independent of the systemic genotoxic stress response.

Peter M Douglas ${ }^{1,2,3}$, Andrew Dillin ${ }^{1,2,3}$

${ }^{1}$ California Institute for Regenerative Medicine, ${ }^{2}$ Howard Hughes Medical Institute, ${ }^{3}$ University of California, Berkeley, CA 94720-3370, USA Correspondence: Andrew Dillin

E-mail: dillin@berkeley.edu

\section{References}

1 Kirkwood TB, Holliday R. Proc R Soc Lond B Biol Sci 1979; 205:531-546.

2 Ermolaeva MA, Segref A, Dakhovnik A, et al. Nature 2013; 501:416-420.

3 Durieux J, Wolff S, Dillin A. Cell 2011; 144:79-91.

4 Taylor RC, Dillin A. Cell 2013: 153:14351447.

5 Prahlad V, Cornelius T, Morimoto RI. Science 2008; 320:811-814.

6 Arantes-Oliveira N, Apfeld J, Dillin A, et al. Science 2002; 295:502-505.

7 Hsin H, Kenyon C. Nature 1999; 399:362366.

8 Vilchez D, Morantte I, Liu Z, et al. Nature 2012; 489:263-268.

9 Gasser S, Orsulic S, Brown EJ, et al. Nature 2005; 436:1186-1190.

10 Gregorio J, Meller S, Conrad C, et al. J Exp Med 2010; 207:2921-2930. 